\title{
Vaccine and COVID-19 Trajectories
}

\author{
Kate H. Choi* \\ Department of Sociology \\ University of Western Ontario \\ ORCID ID: 0000-0001-6885-6233 \\ Patrick A. Denice \\ Department of Sociology \\ University of Western Ontario \\ ORCID ID: 0000-0002-7997-8313 \\ Sagi Ramaj \\ Department of Sociology \\ University of Toronto \\ ORCID ID: 0000-0002-2662-0318
}

July 17, 2021

Manuscript word count including abstract, text, and footnotes: 4,381

Tables: 3

Supplementary table: 1

Corresponding author: Kate H. Choi, Social Science Centre, Room, 5306, London, Ontario,

Canada, N6A 5C2. E-mail: Hchoi228@uwo.ca; Phone: 519-661-2111 x-85115.

Funding acknowledgement: This work was supported by the Social Sciences and Humanities

Research Council (SSHRC) (grant number 430-2017-00920).

Conflict of interest: None

An updated version of this paper was published on October 27, 2021 in Socius

(https://doi.org/10.1177/23780231211052946) 


\section{Vaccine and COVID-19 Trajectories}

Researchers and public health officials posit that vaccine equity holds the key to ending the COVID-19 pandemic. Yet, most prior work on vaccine equity focuses on vaccine hesitancy and seldom compares the vaccine trajectories of neighborhoods with varying COVID-19 levels. Notably scarce are studies that examine the extent to which vaccination helps reduce inequalities in the prevalence of COVID-19 across neighborhoods. Using administrative data from the City of Toronto, we compare the vaccine trajectories of neighborhoods with low, moderate, and high COVID-19 rates. We also examine whether disparities in COVID-19 rates have narrowed or widened as vaccinations have become more available. By mid-June 2021, while differences in vaccination rates by neighborhoods' COVID-19 levels were small, the vaccination rollout has only had a small impact on disparities in COVID-19 rates across neighborhoods. Equality in vaccination rates is not a silver bullet to reducing inequalities in COVID-19 infections across neighborhoods with varying socio-demographic characteristics.

Keywords: COVID-19, vaccines, public health, social determinants, vaccine equity 


\section{Introduction}

Racial minorities, immigrants, and the socio-economically disadvantaged had disproportionately higher COVID-19 infection and fatality rates (Choi et al., 2021; WrigleyField, 2020; Yancy, 2020), yet lower vaccination rates than non-Hispanic Whites and the socioeconomically advantaged (Ndugga et al., 2021). These reports, coupled with the more recent emergence of deadly variants, have kindled concerns about vaccine equity (Jean-Jacques \& Bauchner, 2021). Public health officials have underscored that vaccine equity holds the key to ending the pandemic and have enacted policies to ensure equitable vaccine distribution (Georgieva et al., 2021).

Despite these concerns, few studies have examined whether vaccines are being rolled out equitably. Instead, prior studies focus focused on vaccine hesitancy (for a review, see Sallam, 2021; Viswanath et al., 2021). Vaccine access is another important determinant of uptake. Indeed, recent polls suggest that vaccine hesitancy has decreased the most among African Americans, and differences in vaccine hesitancy by race and income are relatively small (Huetteman, 2021; Maul et al., 2021; Summers, 2021). The few studies on vaccine uptake describe the socio-demographic determinants of vaccine trajectories (Chernyavskiy et al., 2021; Iveniuk \& Leon, 2021). Although these studies offer important insights, they do not explore the extent to which efforts to rollout vaccines more equitably are curbing disparities in COVID-19 infections.

Using administrative data about COVID-19 infection and vaccination rates obtained from the City of Toronto's Open Data Portal, we compare the vaccine trajectories of neighborhoods with low, moderate, and high COVID-19 rates between mid-April and mid-June 2021 — a period when vaccine availability surged in Toronto. We also identify groups who have been left behind 
in the City of Toronto's vaccine rollout. Finally, we examine whether disparities in COVID-19 rates have changed given the vaccine rollout.

\section{Background}

Social determinants of COVID-19

COVID-19 infection and fatalities differ vastly by race/ethnicity, nativity, socioeconomic status, and place of residence (Choi et al., 2021; Rossen et al., 2020; Wrigley-Field et al., 2020; Yancy, 2020). These differences have been attributed to systemic inequalities in occupational attainment, health status, neighborhood contexts, and housing conditions (Bambra et al., 2020; Choi \& Denice, 2020; Krieger et al., 2020; Schneider \& Harknett, 2020).

Of these explanations, three are particularly relevant for our study. First, racial minorities, immigrants, and low-income individuals may not be able to take time off to get vaccinated or to recover from the side effects following vaccination (Schneider \& Harknett, 2020; St-Denis, 2020). Second, immigrants may face language barriers to navigating the health care system and obtaining high-quality care. Limited language proficiency and cultural know-how may also preclude immigrants, particularly recent arrivals, from engaging with the provincial vaccine booking system. Third, these groups more often reside in overcrowded households and dense neighborhoods with fewer health-promoting amenities such as access to family physicians and pharmacies (Bambra et al., 2020; Choi \& Denice, 2020; Kamis et al., 2021; Krieger et al., 2020; Yang et al., 2020). Limited access to health care may mean that racial minorities and low-income individuals may have to travel long distances or wait long periods to get vaccinated. 


\section{Empirical work on COVID-19 vaccination rates}

Prior work on vaccine equity focuses on the socio-demographic determinants of vaccine hesitancy (Sallam, 2021). These studies consistently show that vaccine hesitancy is higher among those who rely on conservative news outlets as their main source of information and have low confidence in scientists (Summers, 2021). Findings of disparities in vaccine hesitancy by race/ethnicity and income are somewhat mixed. Many studies report that non-Hispanic Blacks and the socioeconomically disadvantaged have higher rates of vaccine hesitancy (Carpiano, 2020; Fisher et al., 2020; Nino et al., 2021; Reiter et al., 2020; Sallam, 2021; Viswanath, 2021). Recent polls, however, show that vaccine hesitancy has diminished the most among nonHispanic Blacks and that differences in vaccine hesitancy by race and income are significantly smaller than those along political lines (Summers, 2021).

A small body of work has examined socio-demographic disparities in vaccine uptake. Many public health departments, including the Centers for Disease Control and Prevention and Toronto Public Health, report the vaccine trajectories of different age groups (Centers for Disease Control and Prevention, 2021; City of Toronto, 2021). Chernyavskiy and colleagues (2021) show that vaccines rates were greater in U.S. counties with higher shares of college graduates and racial minorities. Iveniuk and McKenzie (2021) show a negative correlation between previous COVID-19 levels and vaccine rates in Ontario between late March and midApril 2021.

Although these studies offer important insights, they focus solely on vaccine trajectories and do not assess whether vaccination has reduced (or amplified) disparities in COVID-19 rates. Furthermore, Iveniuk and colleagues (2021) examine vaccine uptake in the Province of Ontario between early March and mid-April. This was a time when vaccines were extremely scarce in 
Canada (Lindeman, 2021). Since then, roughly $70 \%$ of Canadians have received at least one dosage of a COVID-19 vaccine, and half of all Canadians aged 12 and over are fully vaccinated (Boynton, 2021; Pelley \& Warren, 2021). After mid-April, the Province of Ontario enacted policies to improve vaccine equity, including allocating more dosages of the COVID-19 vaccine to hotspots (Crawley, 2021; Iveniuk \& McKenzie, 2021). Whether the vaccine inequality observed by Iveniuk and McKenzie (2021) in early April has persisted is unclear. There is a need to systematically address whether vaccine inequities lessened as vaccines became less scarce and the government implemented policies aimed at vaccine equity.

\section{COVID-19 and Vaccination Efforts in City of Toronto}

The context of our study is the City of Toronto in the Province of Ontario, Canada. Canada's COVID-19 death rates fall near the middle of OECD countries, lower than countries like the United States and France, and higher than Japan and Germany (Fallis, 2020). The City of Toronto is a global city with an ethno-racially and socioeconomically diverse population (Social Policy Analysis and Research, 2014). Toronto consists of 140 neighborhoods, comprised of 2-5 census tracts and an average population of 20,000. Neighborhoods in Toronto serve as planning areas for the municipal government, are service areas for local businesses, and are perceived as “actual neighborhoods" by Torontonians (Social Policy Analysis and Research, 2014). Toronto rapidly became a COVID-19 epicenter in Canada, partly reflecting the higher presence of subpopulations hit hard by the COVID-19 pandemic (Choi \& Denice, 2020). COVID-19 rates peaked for the third time in early April 2021 due to the emergence of variants and vaccine scarcity. This peak subsided as vaccines became more readily available in mid-April. By midJune 2021, there have been nearly 170,000 confirmed cases and 3,500 COVID-19-related deaths in the city (City of Toronto, 2021). The City of Toronto experienced a shortage of vaccines until 
mid-April 2021 when just $21 \%$ of adults in Toronto had received at least one dosage. Vaccine supply increased over time, such that by late June 2021 , roughly $70 \%$ of adults in Toronto had received at least one dosage (City of Toronto, 2021).

Two policies were enacted to address vaccine and infection inequalities. First, Ontario prioritized vaccine distribution to older adults and racialized urban neighborhoods (Brown et al., 2021). ${ }^{1}$ For a period of two weeks in early May, half of all new vaccination dosages were allocated to COVID-19 hotspots (Beattie, 2021). Second, unlike other provinces in Canada that did not collect or release COVID-19 data for smaller geographic units, the City of Toronto made publicly available daily COVID-19 infection and death counts, vaccination rates, and sociodemographic profiles for each neighborhood (City of Toronto, 2021). These rich administrative data released by the municipal government make Toronto an ideal setting to analyze issues of vaccine equity in real-time.

\section{Data and Methods}

Data

We combine several pieces of publicly available data. First, daily cumulative vaccination rates for each neighborhood were obtained from the municipal government's COVID-19 vaccination website. Second, information on each neighborhood's socio-demographic composition based on the 2016 Canadian Census was obtained from the City of Toronto's Open Data Catalogue. Third, we obtained the list and locations of pharmacies, hospitals, and clinics administering the COVID-19 vaccines in Toronto. ${ }^{2}$ Our analyses are conducted on the census of the city's neighborhoods $(N=140)$.

\footnotetext{
${ }^{1}$ Provinces are the Canadian equivalent to U.S. states. The Province of Ontario determined the vaccine rollout for the City of Toronto.

${ }^{2}$ Vaccination rate data come from https://www.toronto.ca/home/covid-19/covid-19-latest-city-of-torontonews/covid-19-pandemic-data/covid-19-vaccine-data/. Neighborhood sociodemographic profiles come from:
} 
Measures

We examine two outcomes. First, we measure the cumulative share of adult residents $(18+)$ who have received at least one dose of the COVID-19 vaccine at five time points in the spring and early summer of 2021: (a) April 17, (b) May 1, (c) May 15, (d) May 29, and (e) June 12. These points roughly correspond to major events in the Province of Ontario's vaccination rollout, including changes in age eligibility, increases in the supply of mRNA vaccines, the discontinuation of the use of AstraZeneca for the first dosage due to the risk of developing Vaccine-Induced Prothrombotic Immune Thrombocytopenia (VITT), and the initiation of administration of the second dosage (Ontario, 2021). We focused on first dosages given growing evidence of protection following one of the two dosages of vaccines (Thompson et al., 2021).

Second, we measure the neighborhood-specific cumulative COVID-19 rates as the number of new COVID-19 cases per 100,000 residents through May 1, May 15, May 29, June 12 , and June $23,2021 .^{3}$

Our key independent variable divides Toronto's neighborhoods into terciles based on their cumulative COVID-19 infection rates as of April 1, 2021, when Ontario transitioned into its second and current phase of its vaccination rollout plan. Neighborhoods with low COVID-19 levels refer to those with fewer than 335.59 cumulative COVID-19 cases per 100,000 residents. Neighborhoods with moderate COVID-19 levels had greater than or equal to 355.59, but fewer than 755.16, cumulative COVID-19 cases per 100,000 residents. Neighborhoods with high COVID-19 levels are those with at least 755.16 cumulative COVID-19 cases.

https://www.toronto.ca/city-government/data-researchmaps/open-data/open-data-catalogue. Vaccination administration sites are available at https://www.toronto.ca/home/covid-19/covid-19-protect-yourself-others/covid19-vaccines/covid-19-how-to-get-vaccinated/covid-19-future-city-operated-immunizationclinics/\#location=\&lat=\&lng=.

${ }^{3}$ In supplementary analyses, we modeled the number of cases (instead of rates) and obtained substantively similar results. 
Several time-invariant variables capture the socio-demographic composition of each neighborhood: percent Black, percent South Asian, percent Hispanic, percent East Asian, percent foreign-born, percent who live below the poverty, ${ }^{4}$ percent of essential workers (e.g., health, sales, manufacturing, and education), and percent of adults aged 55 years and older. We opted for this age cut-off because vaccination eligibility was extended to residents under the age of 55 on April 19, 2021 (Ontario, 2021). These variables are standardized (with a mean of 0 and standard deviation of 1 ) to account for their different distributions. ${ }^{5}$

To account for vaccine availability, we note whether a neighborhood included areas designated as COVID-19 hotspots by the provincial government and count the number of vaccine sites in the neighborhood. COVID-19 hotspot is coded 1 if the neighborhood includes areas designated as racialized urban areas (i.e., hotspots) and 0 otherwise. The number of immunization clinics is a count of mass vaccination sites, hospitals, and pharmacies that could administer vaccines. The Ontario government added pharmacies to this list over time. To ensure proper temporal ordering, we relied on the list that we downloaded from the City of Toronto's website in mid-March. For the analyses of COVID-19 trajectories, we also controlled for the vaccination rates on April 17, 2021, and changes in vaccination rates between April 17 and June $23,2021$.

Analytical strategy

In the first stage of the analysis, we estimate two-level growth curve models to compare the vaccine trajectories of neighborhoods with low, moderate, and high COVID-19 rates. Growth

\footnotetext{
${ }^{4}$ Percent of residents whose family income falls below the Low-Income Cut-Offs (LICO) - the Canadian equivalent to the U.S. poverty line.

${ }^{5}$ Models with unstandardized coefficients yield the same results.
} 
curve models estimate differences in COVID-19 vaccination rates in mid-April (i.e., intercepts) and the rate of growth across distinct time points (i.e., slopes) (Curran et al., 2010).

The level-1 equation describes within-neighborhood changes in cumulative vaccination $(n)$ over time $(t)$ :

$$
y_{n t}=a_{n}+\beta_{n t} t+\epsilon_{n t}
$$

Cumulative vaccine rates $\left(y_{n t}\right)$ are characterized by a unique intercept $\left(\alpha_{n}\right)$ and a time-dependent slope $\left(\beta_{n t}\right)$. Time $(t)$ is added as a series of dichotomous variables indicating the day when the cumulative vaccination rates were measured to capture non-linearities in vaccination trajectories.

The level-2 equation models variation in cumulative vaccination infections among low-, middle-, and high-income neighborhoods (i.e., between-neighborhood effects):

$$
\begin{aligned}
& \alpha_{n}=\alpha_{0}+\alpha_{1} x_{n 1}+\alpha_{2} x_{n 2}+\alpha_{3} x_{n 3+}[\ldots]+\alpha_{k} x_{n k}+u_{n} \\
& \beta_{n}=\beta_{0}+\beta_{1} x_{n 1}+\beta_{2} x_{n 2}+\beta_{3} x_{n 3+}[\ldots]+\beta_{k} x_{n k}+v_{n}
\end{aligned}
$$

These equations indicate that the random intercepts $\left(\alpha_{n}\right)$ and slopes $\left(\beta_{n}\right)$ are a function of timeinvariant covariates $\left(x_{n k}\right)$ and error terms $\left(u_{n t}, v_{n t}\right)$.

We estimated three additive models. Model 1 compares the vaccine trajectories of neighborhoods with varying levels of COVID-19 levels in the absence of any controls. Model 2 accounts for differences in vaccine availability by adding hotspot status and the number of vaccination sites in the neighborhoods. Model 3 adds socio-demographic controls to gain insights into the vaccine trajectories of different groups and identify the groups being left behind in Toronto's vaccine rollout.

In the second stage of the analysis, we estimate negative binomial regression models comparing the number of new COVID-19 cases (per 100,000 residents) in neighborhoods with varying levels of COVID-19. We ran analogous growth curve models as in the first stage to 
predict the COVID-19 trajectories between early May and late June. The only noteworthy difference is that Models 2 and 3 also include vaccination rates in mid-April 2021 and changes in vaccination rates between mid-April and end-June 2021. The variance inflation factors (VIFs) of all estimated models are less than 4 . We also accounted for clustering by neighborhood.

\section{Results}

\section{Sample characteristics}

Table 1 documents variation in the socio-demographic composition of neighborhoods by their level of COVID-19 rates. Consistent with prior work (Choi et al., 2021; Wrigley-Field et al., 2020), neighborhoods with higher COVID-19 rates are more ethno-racially diverse than neighborhoods with lower COVID-19 levels. For example, 14\% of residents of neighborhoods with high COVID-19 rates self-identify as Black, compared to $9 \%$ of residents of neighborhoods with moderate COVID-19 rates and 4\% of residents of neighborhoods with low COVID-19 rates. Nearly $60 \%$ of residents of neighborhoods with high COVID-19 rates are foreign-born, compared to $50 \%$ of residents of neighborhoods with moderate COVID-19 rates and 37\% of residents of neighborhoods with low COVID-19 rates. They are also more socioeconomically disadvantaged than neighborhoods with lower COVID-19 rates. Thirty-one percent of residents of neighborhoods with high COVID-19 rates were college graduates, compared to $43 \%$ of residents of neighborhoods with moderate and $57 \%$ of residents of neighborhoods with low COVID-19 rates. Higher shares of residents of neighborhoods with high COVID-19 rates are essential workers: 52\% in neighborhoods with high COVID-19 rates versus 44-47\% in other neighborhoods. They are also more likely to include areas designated as COVID-19 hotspots and host more vaccination sites.

Table 1 goes here. 


\section{Growth Curve Models Predicting Vaccination Trajectories}

Table 2 presents the results from growth curve models predicting the cumulative percentage of adults who got at least one dosage by neighborhood COVID-19 levels. The main effects $(\alpha)$ capture the differences in cumulative vaccination rates in mid-April 2021, and the slopes $(\beta)$ capture the differences in changes in vaccination rates over time. We obtain estimates based on the census of neighborhoods in Toronto; thus, our interpretations are based on the size of the coefficient and substantive importance. ${ }^{6}$

\section{Table 2 goes here.}

In Model 1, vaccination rates were lower in neighborhoods with higher cumulative COVID-19 rates. By mid-April 2021, the cumulative share of adults in neighborhoods with moderate and high COVID-19 rates were, respectively, 4.4 and 5.8 percentage points lower than the corresponding share for neighborhoods with low COVID-19 rates. However, vaccination rates then grew faster in neighborhoods with higher COVID-19 rates. By mid-June 2021, these differences had narrowed to 1.0 and 1.7 percentage points.

Model 2 adds hotspot status and the number of vaccine sites. Until mid-April 2021, vaccine rates were lower in neighborhoods with COVID-19 hotspots and more vaccination sites. Between mid-April and mid-June 2021, vaccination rates grew faster in neighborhoods with COVID-19 hotspots. Vaccination rates initially grew slower in neighborhoods with more vaccination sites, but starting in mid-May 2021, they grew faster. Disparities in vaccine access explained little of the initial differences in COVID-19 vaccination rates across neighborhoods. Net of controls for COVID-19 hotspot status and the number of vaccination sites, differences in

\footnotetext{
${ }^{6}$ Statistical significance is used to test whether the difference between sample means is more or less equal to the population mean. We are running our analyses for all 140 neighborhoods in the city, and so the coefficients capture the population estimates.
} 
the growth rate between neighborhoods with low and moderate COVID-19 rates decreased by $30 \%:\left[100 * \frac{(2.90-2.17)}{2.90}=30\right]$. Differences in growth rate between neighborhoods with low and high COVID-19 rates diminished by $20 \%:\left[100 * \frac{(4.13-3.31)}{4.13}=20\right]$.

Model 3 adds socio-demographic traits. COVID-19 vaccination rates were lower in neighborhoods with high population density and higher shares of foreign-born and Indigenous residents. By contrast, COVID-19 vaccination rates were higher in neighborhoods with higher shares of residents who are ages 55 years and older, essential workers, and college graduates. COVID-19 vaccination rates increased faster in neighborhoods with higher shares of South Asians, East Asian, and Indigenous residents, COVID-19 hotspots, and higher population density. By contrast, they increased at a slower pace in low-income and immigrant neighborhoods. Due to lower initial rates and slower rates of growth, vaccination rates were exceptionally low in neighborhoods with high shares of foreign-born residents. By mid-June 2021, a standard deviation increase in the share of foreign-born residents was associated with a vaccination rate that was roughly five percentage points lower.

Net of socio-demographic controls, COVID-19 vaccination rates in mid-April would have been higher in neighborhoods with moderate and high COVID-19 rates than in neighborhoods with low COVID-19 rates. Similarly, socio-demographic controls explain roughly $75-80 \%$ of the growth rates between neighborhoods with low COVID-19 rates and other communities. These findings attest to high initial barriers to vaccine access in neighborhoods with higher COVID-19 rates and larger racial minority, immigrant, and low-income populations, but these barriers become less salient over time.

Growth Curve Models Predicting Cumulative COVID-19 Rates 
Table 3 presents results from growth curve models predicting the cumulative number of COVID-19 cases per 100,000 residents. Model 1 presents the zero-order association between infection trajectories of neighborhoods with a varying initial prevalence of COVID-19. Although all communities experienced a rise in COVID-19 cases, COVID-19 infections spread faster in neighborhoods with a higher prevalence of COVID-19 infections. Whereas neighborhoods with a low prevalence of COVID-19 cases saw 341 more infections, moderate and high neighborhoods increased their number of infections by 572 and 762 , respectively.

Model 2 controls for vaccine availability and vaccination rates. In early May 2021, the cumulative COVID-19 rates were higher in COVID-19 hotspots, and COVID-19 infections grew faster in these communities. By contrast, a higher vaccination rate was associated with lower cumulative infections in early May and slower infection growth between early May and late June. The same pattern obtains for neighborhoods with more vaccination sites and a more rapid rise in vaccination rates. Vaccine availability and uptakes, however, explain little of the differences in the growth in COVID-19 rates by neighborhood COVID-19 levels.

In Model 3, COVID-19 infections spread at a slower pace in neighborhoods with higher shares of East Asians, Blacks, and college graduates, and faster in neighborhoods with higher shares of residents who were born abroad and essential workers. Controlling for differences in socio-demographic traits explains $67 \%$ of the differences in initial COVID-19 cases between neighborhoods with low and moderate COVID-19 levels and 75\% of the difference in initial COVID-19 cases between neighborhoods with low and high COVID-19 levels. It also explains over $90 \%$ of the difference in pace of growth in COVID-19 cases by the neighborhood prevalence of COVID-19 rates between early May and late June. These findings suggest that differences in the socio-demographic characteristics of neighborhoods and their residents' 
differential risk of contracting COVID-19 are key factors behind disparities in COVID-19 rates across communities.

\section{Discussion}

Researchers and public health officials posit that equitable vaccine access is the best hope for defeating the COVID-19 pandemic. Our study (a) compares the vaccine trajectories of neighborhoods with varying COVID-19 levels in a period before vaccination was widespread in Toronto and (b) describes whether differences in COVID-19 trajectories have changed as a higher share of residents of Toronto have become vaccinated over time.

Consistent with prior work, we show that, in mid-April 2021, vaccination rates were lower in neighborhoods in Toronto with higher COVID-19 rates (Iveniuk \& Leon, 2021). Vaccination rates, however, increased at a faster pace in these neighborhoods over time. Vaccine availability explained $20-30 \%$ of the differences in vaccination rates across neighborhoods with varying COVID-19 levels. These findings suggest that the prioritization of communities with high COVID-19 rates may be an effective way to achieve parity in vaccination rates.

Nonetheless, it is possible that the municipal government started to prioritize vaccination availability in racialized urban communities after mid-April as vaccine supply increased. In this way, barriers to vaccination among the disadvantaged may have decreased as vaccines became less scarce and parity in vaccination rates would have partially occurred even in the absence of such policies.

Differences in vaccination trajectories across neighborhoods with varying COVID-19 levels emerge in large part due to the socio-demographic traits of their residents. Neighborhoods with higher shares of college graduates, essential workers, and older adults had higher initial vaccination rates. By contrast, neighborhoods with higher shares of immigrant and Indigenous 
residents had lower initial vaccination rates. In most cases, vaccination rates grew at a slower pace for those who had high initial vaccination rates and faster for those with low initial vaccination rates. An exception was found in neighborhoods with high shares of immigrants. They had both lower initial vaccine rates and a slower pace of growth. Policymakers should pay greater attention to these neighborhoods that may have been left behind in their vaccination efforts.

Despite having achieved high and nearly equal vaccination rates, neighborhoods in Toronto with higher COVID-19 levels continued to experience significantly more infections. Vaccine availability explained little of the differences in new COVID-19 cases among neighborhoods with varying COVID-19 rates. Accounting for differences in socio-demographic composition, however, explained all of these disparities. Overall, this suggests that differences in COVID-19 rates emerge in large part due to variation in the socio-demographic makeup of residents who have differential exposure to the COVID-19 virus. In the absence of universal vaccination rates, achieving equal vaccination rates will not reduce disparities in COVID-19 rates. To ensure true equity, residents of neighborhoods with higher COVID-19 rates may have to be vaccinated at higher rates to account for their greater exposure to the COVID-19 virus. Furthermore, vaccination rollouts have to work in tandem with other efforts aimed at reducing exposure to the COVID-19 virus, such as the enforcement of safety measures to curb the COVID-19 viruses in some worksites (Su et al., 2021).

Like prior work, our paper has some limitations. First, we have access to neighborhoodlevel vaccination rates. Given recent polls revealed that vaccine hesitancy differs little by race and income levels (Summers, 2021), we attribute disparities in vaccination rates largely to differential barriers to access. Although are some media reports that show substantial decline in 
vaccination rates among Black Torontonians (McGillivray, 2021), the extent to which these disparities emerge due to vaccine hesitancy needs to be formally tested for the City of Toronto. Second, information on place of residence was missing for $7.7 \%$ of Toronto residents who received at least one dosage of the COVID-19 vaccine. We thus understate the share of Torontonians who have received at least one dosage; however, whether missing rates are higher for residents of neighborhoods with higher COVID-19 is unknown. Third, we do not have access to data about the number of daily first dosages that were made available to each neighborhood. We indirectly captured vaccine supply using (a) the designation as a COVID-19 hotspot (the Province of Ontario allocated more vaccines to these urban racialized communities) and (b) the number of vaccine sites in the neighborhood. Fourth, neighborhood boundaries delineated by the City of Toronto do not align perfectly with the boundaries used by the Province of Ontario to designate hotspots (i.e., forward sortation areas). We operationalized COVID-19 hotspots as neighborhoods that included an area that was designated as a COVID-19 hotspot. Therefore, unknown is what share of the residents of these neighborhoods had faster access to vaccines given their residence in a hotspot. Nonetheless, it is worth noting that alternate operationalizations of COVID-19 hotspots yielded similar results. Similarly, our measure of number of vaccine sites may have not adequately captured vaccine supply at various points in time. Before mid-April, Canada experienced severe vaccine shortages (Lindeman, 2021). Furthermore, the Province of Ontario suspended the administration of the AstraZeneca vaccine for the first dosages in May 2021. COVID-19 vaccines may have sometimes not been readily available in each COVID-19 vaccine site. Finally, the emergence of variants with higher infectiousness may have canceled out the impact of vaccination in reducing COVID-19 
disparities across neighborhoods. Future forecasting models should make this assessment when the requisite data becomes available.

Our study highlights the fact that fully achieving vaccine equity may require more than simple parity in vaccination availability and rates. Instead, it may entail that we develop a more nuanced understanding of the structural conditions of neighborhoods and design a more comprehensive strategy to protect the vulnerable from exposure to the COVID-19 virus. Doing so may be our best weapon to defeat the COVID-19 epidemic. 


\section{References}

Bambra, C., R. Riordan, J. Ford, \& F. Matthews. 2020. “The COVID-19 pandemic and health inequalities”. Journal of Epidemiology \& Community Health 74(11), 964-968. http://dx.doi.org/10.1136/jech-2020-214401

Beattie, S. 2021. "Ontario stops increased vaccine supply to Toronto hotspots as city officials urge province to reconsider". $C B C$, May 12, https://www.cbc.ca/news/canada/toronto/toronto-covid-update-may-12-1.6023534

Boynton, S. 2021. “Over 50\% of eligible Canadians now fully vaccinated against COVID-19 as cases fall”. Global News, July 12, https://globalnews.ca/news/8022588/covid-canada$\underline{\text { cases-vaccinations-july-12-2021 }}$

Brown, K. A., N. Stall, E. Joh, U. Allen, I. Bogoch, S.A. Buchan, N. Daneman, G.A. Evans, D. Fisman, J. L. Gibson, J. L., J. Hopkins, T. Van Ingen, A. Maltsev, A. McGeer, S. Mishra, S., F. Razak, B. Sander, B. Schwartz, K, Schwartz, ... P. Jüni, 2021. “A strategy for the mass distribution of COVID-19 vaccines in Ontario based on age and neighbourhood". Science Briefs of the Ontario COVID-19 Science Advisory Table, 2(10), 1-16. https://doi.org/10.47326/ocsat.2021.02.10.1.0

Carpiano, R. M. 2020. “Demographic differences in U.S. adult intentions to receive a potential coronavirus vaccine and implications for ongoing study". MedRxiv. https://doi.org/10.1101/2020.09.07.20190058

Centers for Disease Control and Prevention. 2021. United States COVID-19 cases, deaths, and laboratory testing (NAATs) by state, territory, and jurisdiction. Retrieved from https://covid.cdc.gov/covid-data-tracker/\#cases_casesper100k. Accessed July 14, 2021. 
Chernyavskiy, P., J. W. Richardson, and S. J. Ratcliffe. 2021. COVID-19 vaccine uptake in United States counties: Geospatial vaccination patterns and trajectories towards herd immunity. MedRxiv. https://doi.org/10.1101/2021.05.28.21257946

Choi, K. H., P. Denice, M. Haan, and A. Zajacova. 2021. "Studying the social determinants of COVID-19 in a data vacuum". Canadian Review of Sociology, 58(2), 146-164. https://doi.org/10.1111/cars.12336

Choi, K. H., and P. Denice. 2020. "Neighborhood SES and the COVID-19 pandemic". SocArXiv. https://doi.org/10.31235/osf.io/3xg5q

City of Toronto. 2021. COVID-19: Vaccine data. Retrieved from https://www.toronto.ca/home/covid-19/covid-19-latest-city-of-toronto-news/covid-19pandemic-data/covid-19-vaccine-data. Accessed July 14, 2021.

Crawley, M. 2021. “These 'hot spots' getting vaccine priority are less hard-hit by COVID-19 than Ontario average". CBC News, April 12. https://www.cbc.ca/news/canada/toronto/ontario$\underline{\text { covid-19-vaccination-postal-code-hot-spots-1.5983155 }}$

Curran, P. J., K. Obeidat, and D. Losardo. 2010. "Twelve frequently asked questions about growth curve modeling”. Journal of Cognition and Development, 11(2), 121-136. https://doi.org/10.1080/15248371003699969

Fallis, G. 2020. “2020 hindsight — George Fallis: Scoring Canada's COVID fight over its first three rounds of six". Financial Post. December 31. https://financialpost.com/opinion/2020-hindsight-george-fallis-scoring-canadas-covidfight-over-its-first-three-rounds-of-six 
Fisher, K. A., S. Bloomstone, J. Walder, S. Crawford, H. Fouayzi, and K. Mazor. 2020.

“Attitudes toward a potential SARS-CoV-2 vaccine: A survey of U.S. Adults". Annals of Internal Medicine, 173(12), 964-973. https://doi.org/10.7326/M20-3569

Georgieva, K., T. A. Ghebreyesus, D. Malpass, and N. Okonjo-Iweala. 2021. “A new commitment for vaccine equity and defeating the pandemic". World Health Organization, May 31. https://www.who.int/news-room/commentaries/detail/a-new-commitment-forvaccine-equity-and-defeating-the-pandemic

Huetteman, E. 2021. "Covid vaccine hesitancy drops among all Americans, new survey shows". Kaiser Health News, March 30. https://khn.org/news/article/covid-vaccine-hesitancydrops-among-americans-new-kff-survey-shows/

Iveniuk J., and S. Leon. 2021. "An uneven recovery: Measuring COVID-19 vaccine equity in Ontario". Wellesley Institute. https://www.wellesleyinstitute.com/wpcontent/uploads/2021/04/An-uneven-recovery-Measuring-COVID-19-vaccine-equity-in$\underline{\text { Ontario.pdf }}$

Iveniuk J., and K. McKenzie. 2021. "Has the advice to increase vaccination in hot spots improved equity in Ontario?’. Wellesley Institute. https://www.wellesleyinstitute.com/wp-content/uploads/2021/05/Has-the-advice-toincrease-vaccination-in-hot-spots-improved-equity-in-Ontario.pdf

Jean-Jacques, M. and H. Bauchner. 2021. “Vaccine distribution-Equity left behind?”. JAMA: The Journal of the American Medical Association, 325(9), 829-830. https://doi.org/10.1001/jama.2021.1205

Kamis, C., A. Stolte, J. West, S. Fishman, T. Brown, and H. Farmer. 2021. “Overcrowding and COVID-19 mortality across U.S. counties: Are disparities growing over time?’. Social 
Science \& Medicine - Population Health, 15, Article 100845.

https://doi.org/10.1016/j.ssmph.2021.100845

Krieger, N., P. Waterman, and J. T. Chen. 2020. "COVID-19 and overall mortality inequities in the surge in death rates by zip code characteristics: Massachusetts, January 1 to May 19, 2020". American Journal of Public Health, 110(12), 1850-1852.

https://doi.org/10.2105/AJPH.2020.305913

Lindeman, T. 2021. Canada's vaccine mess. The Atlantic April 6.

https://www.theatlantic.com/international/archive/2021/04/canada-vaccine-rolloutproblems $/ 618516 /$

Maul, A., K. Reddy, and M. Joshi. 2021. Vaccine equity index shows reduction in Maryland COVID-19 vaccination disparity in less than two months. NEJM Catalyst: Innovations in Care Delivery. https://catalyst.nejm.org/doi/full/10.1056/CAT.21.0126

McGillivray, K. 2021. 20 townhalls later, here's how Toronto's Black scientists' task force reduced vaccine hesitancy. CBC News, June 15.

https://www.cbc.ca/news/canada/toronto/20-townhalls-later-here-s-how-toronto-s-black$\underline{\text { scientists-task-force-reduced-vaccine-hesitancy-1.6064806 }}$

Ndugga, N., O. Pham, L. Hill, S. Artiga and N. Parker. 2021. "Latest data on COVID-19 by race/ethnicity". Kaiser Family Foundation, July 8. https://www.kff.org/coronaviruscovid-19/issue-brief/latest-data-on-covid-19-vaccinations-race-ethnicity/

Niño, M. D., B. Hearne, and T. Cai. 2021. Trajectories of COVID-19 vaccine intentions among U.S. adults: The role of race and ethnicity. Social Science \& Medicine - Population Health, 15, Article 100824. https://doi.org/10.1016/j.ssmph.2021.100824 
Ontario. 2021. Ontario's COVID-19 vaccination plan. Retrieved from https://covid19.ontario.ca/ontarios-covid-19-vaccination-plan. Accessed July 14, 2021.

Pelley, L., and E. Warren. 2021. "Canada now among top countries for 1st doses of COVID-19 vaccines. So how high can we go?" CBC News, June 3. https://www.cbc.ca/news/health/canada-now-among-top-countries-for-1st-doses-of$\underline{\text { covid-19-vaccines-so-how-high-can-we-go-1.6050268 }}$

Reiter, P. L., M. Pennell, and M. L. Katz. 2020. “Acceptability of a COVID-19 vaccine among adults in the United States: How many people would get vaccinated?". Vaccine, 38(42), 6500-6507. https://doi.org/10.1016/j.vaccine.2020.08.043

Rossen, L. M., A. M. Branum, F. B. Ahmad, P. Sutton, and R.N. Anderson. 2020. “Excess deaths associated with COVID-19, by age and race and ethnicity — United States, January 26-October 3, 2020”. Morbidity \& Mortality Weekly Report, 69(42), 1522-1527. http://dx.doi.org/10.15585/mmwr.mm6942e2

Sallam, M. 2021. "COVID-19 vaccine hesitancy worldwide: A concise systematic review of vaccine acceptance rates". Vaccines, 9(2), Article 160. https://doi.org/10.3390/vaccines9020160

Schneider, D., and K. Harknett. 2020. "Essential and unprotected: COVID-19-related health and safety procedures for service-sector workers". Shift Research Briefs. https://shift.hks.harvard.edu/essential-and-unprotected-covid-19-related-health-andsafety-procedures-for-service-sector-workers/

Social Policy Analysis and Research. 2014. TSNS 2020 neighbourhood equity index: Methodological documentation. City of Toronto. https://www.toronto.ca/legdocs/mmis/2014/cd/bgrd/backgroundfile-67350.pdf 
St-Denis, X. 2020. "Sociodemographic determinants of occupational risks of exposure to COVID-19 in Canada”. Canadian Review of Sociology, 57(3), 399-452. https://doi.org/10.1111/cars.12288

Su, Z., J. Wen, D. McDonnell, E. Goh, X. Li, S. Šegalo, J. Ahmad, A. Cheshmehzangi, and Y. Xiang 2021. "Vaccines are not yet a silver bullet: The imperative of continued communication about the importance of COVID-19 safety measures". Brain, Behavior, \& Immunity - Health, 12, Article 10024. https://doi.org/10.1016/j.bbih.2021.100204

Summers, J. 2021. Little difference in vaccine hesitancy among white and black Americans, poll finds. National Public Radio, March 12. https://www.npr.org/sections/coronavirus-liveupdates/2021/03/12/976172586/little-difference-in-vaccine-hesitancy-among-white-andblack-americans-poll-find

Thompson, M. G., J. L. Burgess, A.L. Naleway, H. L. Tyner, S.K. Yoon, J. Meece, L. Olsho, A. Caban-Martinez, A. Fowlkes, K. Lutrick, J. L. Kuntz, K. Dunnigan, M. J. Odean, K. Hegmann, E. Stefanski, L. Edwards, N. Schaefer-Solle, L, Grant, K. Ellingson, ... M. Gaglani 2021. "Interim estimates of vaccine effectiveness of BNT162b2 and mRNA1273 COVID-19 vaccines in preventing SARS-CoV-2 infection among health care personnel, first responders, and other essential and frontline workers — Eight U.S. locations, December 2020-March 2021”. Morbidity \& Mortality Weekly Report, 70(13), 495-500. http://dx.doi.org/10.15585/mmwr.mm7013e3

Viswanath, K., M. Bekalu, D. Dhawan, R. Pinnamaneni, J. Lang, and R. McLoud. 2021. "Individual and social determinants of COVID-19 vaccine uptake". BMC Public Health, 21, Article 818. https://doi.org/10.1186/s12889-021-10862-1 
Wrigley-Field, E., S. Garcia, J. Leider, C. Robertson, R. Wurtz. 2020. "Racial disparities in COVID-19 and excess mortality in Minnesota". Socius: Sociological Research for a Dynamic World, 6, 1-4. https://doi.org/10.1177/2378023120980918

Yancy, C. W. 2020. COVID-19 and African Americans. JAMA: The Journal of the American Medical Association, 323(19), 1891-1892. https://doi.org/10.1001/jama.2020.6548

Yang, J., K. Allen, R. Mendleson, and A. Bailey. 2020. Toronto's COVID-19 divide: The city's northwest corner has been 'failed by the system'. Toronto Star, , June 28. https://www.thestar.com/news/gta/2020/06/28/torontos-covid-19-divide-the-citysnorthwest-corner-has-been-failed-by-the-system.html 


\section{TABLES}

Table 1. Sample characteristics

\begin{tabular}{lccc}
\hline & High & Moderate & Low \\
\cline { 2 - 4 } & 46 & 48 & 46 \\
\hline A. Socio-demographic traits & & & \\
\% Black & 13.8 & 8.6 & 4.0 \\
\% South Asian & 17.9 & 9.9 & 4.8 \\
\% East Asian & 17.9 & 18.7 & 14.2 \\
\% Hispanic & 4.1 & 3.2 & 1.7 \\
\% Foreign-born & 58.6 & 50.4 & 37.1 \\
\% Neither English/French & 5.9 & 4.9 & 2.9 \\
\% Aboriginal & 0.7 & 0.9 & 1.1 \\
\% College graduate & 31.3 & 42.5 & 57.0 \\
\% 55+ years of age & 28.0 & 28.1 & 29.6 \\
\% Below the poverty line & 19.2 & 17.5 & 14.0 \\
\% Essential worker & 51.9 & 47.2 & 43.6 \\
Vaccine availability & & & \\
Hot spot & 45.7 & 14.6 & 6.5 \\
\# Vaccination sites & 2.1 & 2.0 & 1.4 \\
Population density & 5,694 & 6,853 & 6.211 \\
\hline
\end{tabular}

Source: The City of Toronto's Open Data Source

Notes: Analyses are collected on all 140 neighborhoods in the City of Toronto. 
Table 2. Growth Curve Models Predicting Vaccination Rates by Neighborhood COVID-19 Rates

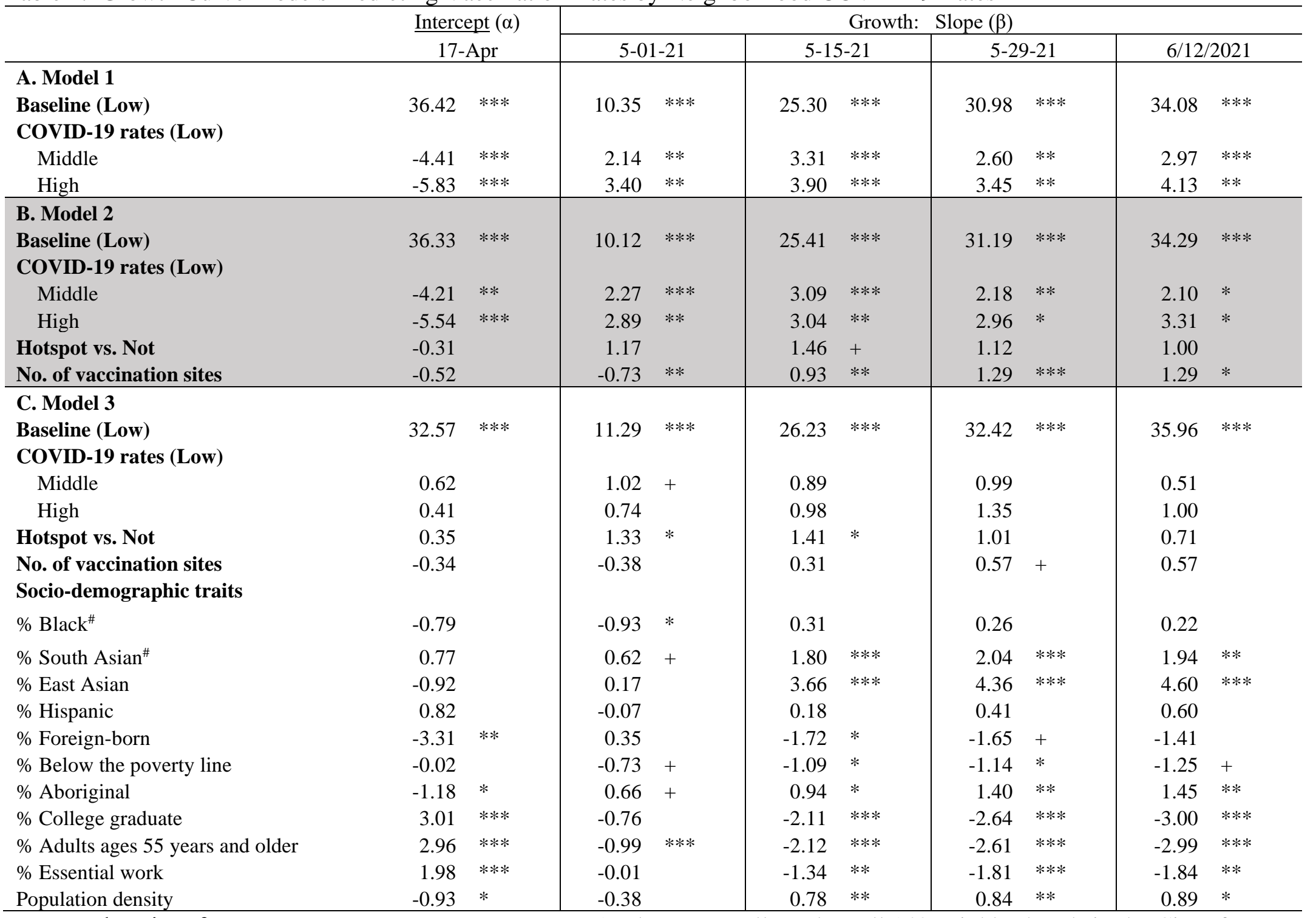

Source: The City of Toronto's Open Data Source. Notes: Analyses are collected on all 140 neighborhoods in the City of Toronto. 
Table 3. Growth Curve Models Predicting COVID-19 Trajectories by Neighborhood COVID-19 Rates

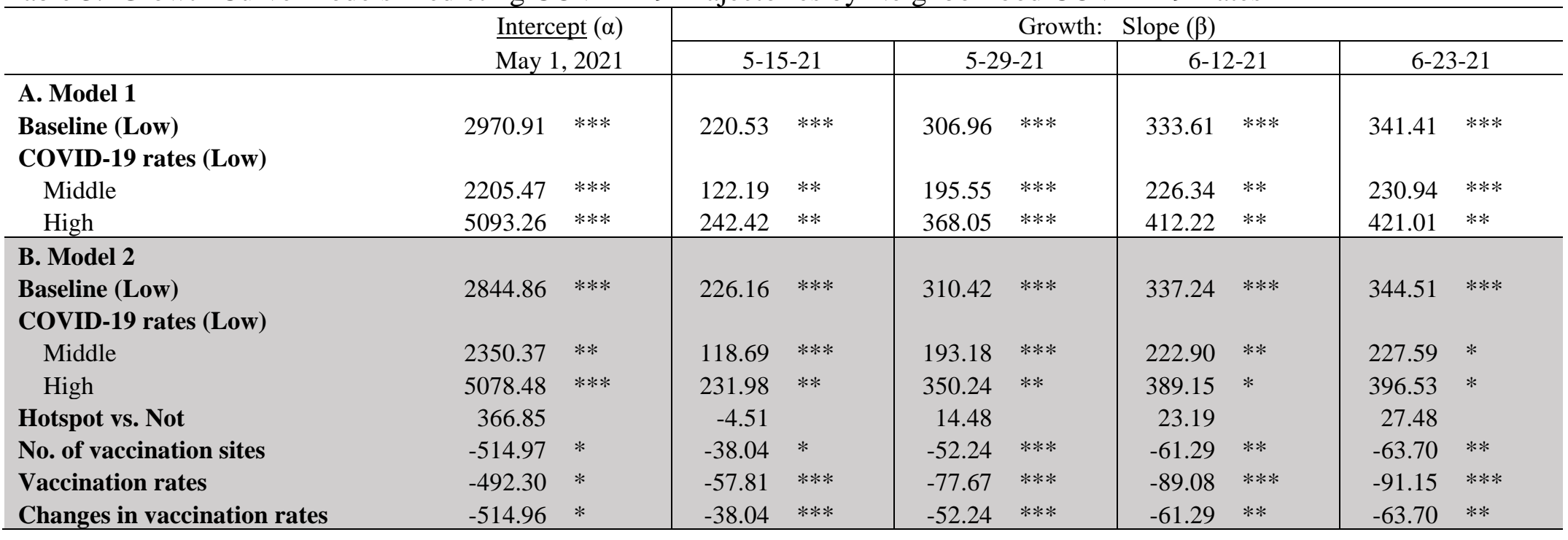


Table 3. (Continued)

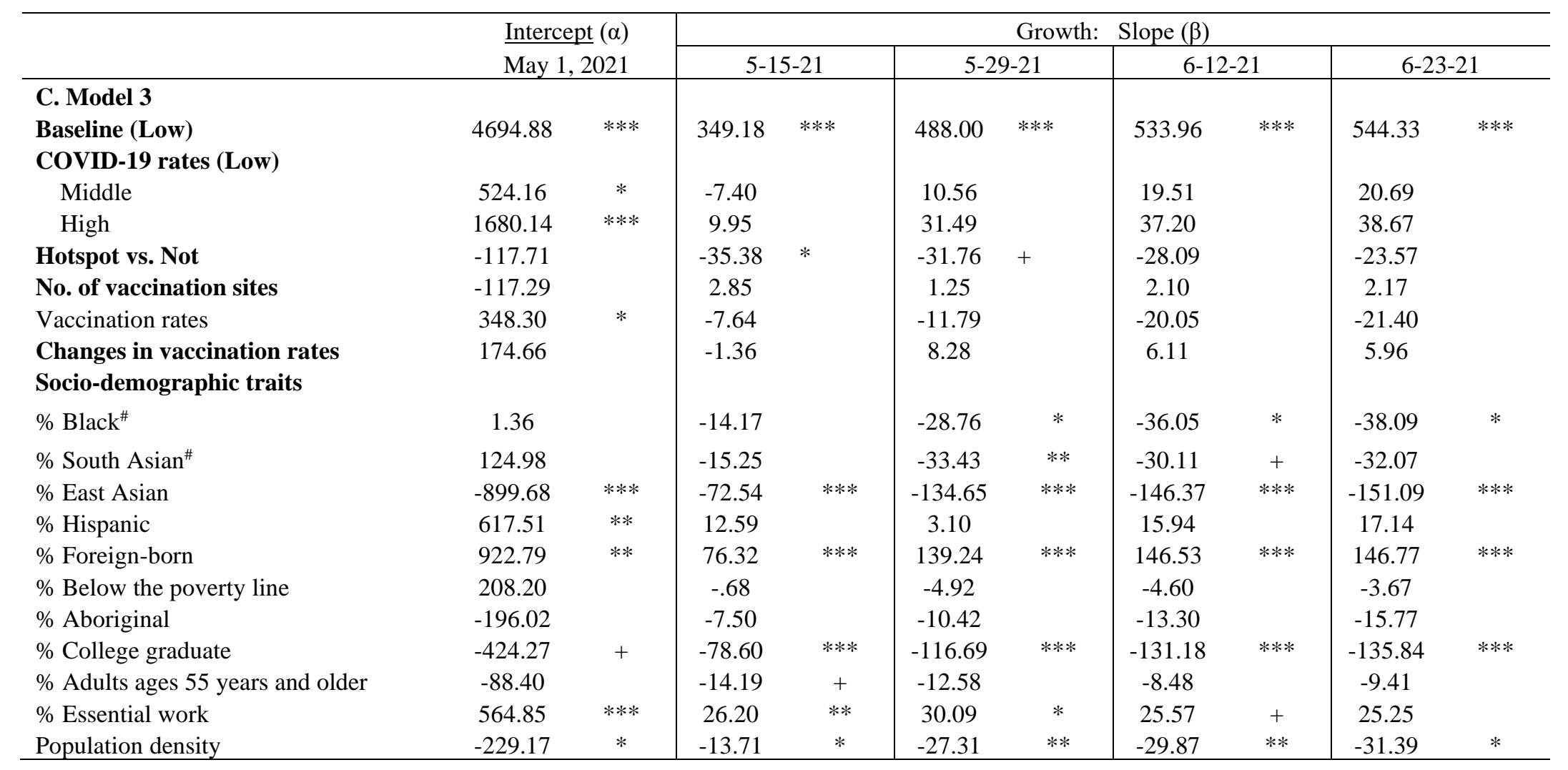

Source: The City of Toronto's Open Data Source. Notes: Analyses are collected on all 140 neighborhoods in the City of Toronto 


\section{$\underline{\text { Appendix }}$}

Table A1. Vaccination Rates, New COVID-19 rates, and Cumulative COVID-19 rates

\begin{tabular}{lccc}
\hline & High & Moderate & Low \\
\cline { 2 - 4 } & 46 & 48 & 46 \\
\hline Vaccination rates & & & \\
17-Apr-21 & 30.6 & 32.0 & 36.4 \\
01-May-21 & 44.3 & 44.5 & 46.8 \\
15-May-21 & 58.9 & 59.9 & 61.7 \\
29-May-21 & 65.5 & 66.4 & 67.4 \\
12-Jun-21 & 68.8 & 69.5 & 70.5 \\
New COVID-19 rates & & & \\
01-May-21 & 49.1 & 35.4 & 19.4 \\
15-May-21 & 24.7 & 17.7 & 9.4 \\
29-May-21 & 7.2 & 4.8 & 3.4 \\
12-Jun-21 & 3.1 & 2.5 & 0.8 \\
23-Jun-21 & 0.1 & 0.0 & 0.0 \\
Cumulative COVID-19 rates & & & \\
01-May-21 & 8064.2 & 5176.4 & 2970.9 \\
15-May-21 & 8527.1 & 5519.1 & 3191.4 \\
29-May-21 & 8739.2 & 5678.9 & 3277.9 \\
12-Jun-21 & 8810.0 & 5736.3 & 3304.5 \\
23-Jun-21 & 8826.6 & 5748.7 & 3312.3 \\
\hline
\end{tabular}

